\title{
Nonperiodic Synchronization in Heterogeneous Networks of Spiking Neurons
}

\author{
Jean-Philippe Thivierge ${ }^{1}$ and Paul Cisek ${ }^{2}$ \\ ${ }^{1}$ Department of Psychological and Brain Sciences, Indiana University, Bloomington, Indiana 47405, and ²Groupe de Recherche sur le Système Nerveux \\ Central, Département de Physiologie, Université de Montréal, Montréal, Québec, Canada H3T 1J4
}

\begin{abstract}
Neural synchronization is of wide interest in neuroscience and has been argued to form the substrate for conscious attention to stimuli, movement preparation, and the maintenance of task-relevant representations in active memory. Despite a wealth of possible functions, the mechanisms underlying synchrony are still poorly understood. In particular, in vitro preparations have demonstrated synchronization with no apparent periodicity, which cannot be explained by simple oscillatory mechanisms. Here, we investigate the possible origins of nonperiodic synchronization through biophysical simulations. We show that such aperiodic synchronization arises naturally under a simple set of plausible assumptions, depending crucially on heterogeneous cell properties. In addition, nonperiodicity occurs even in the absence of stochastic fluctuation in membrane potential, suggesting that it may represent an intrinsic property of interconnected networks. Simulations capture some of the key aspects of population-level synchronization in spontaneous network spikes (NSs) and suggest that the intrinsic nonperiodicity of NSs observed in reduced cell preparations is a phenomenon that is highly robust and can be reproduced in simulations that involve a minimal set of realistic assumptions. In addition, a model with spike timing-dependent plasticity can overcome a natural tendency to exhibit nonperiodic behavior. After rhythmic stimulation, the model does not automatically fall back to a state of nonperiodic behavior, but keeps replaying the pattern of evoked NSs for a few cycles. A cluster analysis of synaptic strengths highlights the importance of population-wide interactions in generating this result and describes a possible route for encoding temporal patterns in networks of neurons.
\end{abstract}

Key words: spontaneous activity; synchronization; spike timing-dependent plasticity; network spike; tetanization; integrate-and-fire neurons

\section{Introduction}

Like musicians performing a classical symphony, individual cells of the cortex act in close interaction with one another and have the ability to precisely orchestrate their neural activity to control behavior (Riehle et al., 1997). An important feature of this interaction is synchronization of neural activity, whereby populations of cells fire in close temporal contiguity (Marom and Shahaf, 2002). Although several functional roles of synchronization have been proposed, including visual system development (Feller et al., 1997), conscious attention to stimuli (Melloni et al., 2007), movement preparation (Riehle et al., 1997), and the maintenance of representations in memory (Axmacher et al., 2006), the precise neural mechanisms responsible are still unclear. In particular, it remains unexplained how spontaneous neural activity (i.e., not directly linked to environmental input) occurs in a nonperiodic manner (i.e., at intervals that are not regular) (Elbert et al., 1994)

Received Feb. 27, 2008; revised June 24, 2008; accepted June 26, 2008.

This work was supported by a postdoctoral fellowship from the Fonds Québéçois de Recherche sur la Nature et les Technologies, a research grant from the National Science and Engineering Research Council, and an infrastructure grant from the Fonds de Recherche en Santé du Québec. We are thankful to Rob de Ruyter van Steveninck, John M. Beggs, Jeff L. Krichmar, and two anonymous reviewers for comments.

Correspondence should be addressed to Dr. Jean-Philippe Thivierge, Department of Psychological and Brain Sciences, 1101 East Tenth Street, Bloomington, IN 47405. E-mail: jthivier@indiana.edu.

D0I:10.1523/JNEUROSC1.0870-08.2008

Copyright $\odot 2008$ Society for Neuroscience $\quad$ 0270-6474/08/287968-11\$15.00/0 and with long temporal intervals (i.e., 1-2 Hz) (Eytan and Marom, 2006). This lack of periodicity cannot be explained by simple oscillatory mechanisms, including models of neural dynamics whose energy function settles in fixed point attractors such as a stable synchronous state (Gerstner and Kistler, 2002) or a limit cycle (Rabinovich and Abarbanel, 1998).

Here, we investigate the possible origins of nonperiodic synchronization through biophysical simulations aimed at determining whether aperiodic network spikes (NSs), forming temporally precise $(<100 \mathrm{~ms})$ events of synchronization, arise under a basic set of plausible assumptions. The model consists of a population of interconnected excitatory integrate-and-fire neurons, each of which possesses different intrinsic properties, including integration rates and spike thresholds. This "cellular heterogeneity" is consistent with experimental evidence supporting the idea that, in populations of intercommunicating neurons, different cells possess different levels of excitability (Murthy et al., 1997) and discharge patterns (Bland et al., 2005).

In this study, we propose that cellular heterogeneity contributes strongly to the nonperiodic nature of synchronization observed experimentally. In addition, we aim to show that the lack of periodicity in the model does not prevent it from generating more periodic synchronization in response to external stimulation (Bracci et al., 1999; Mikkonen et al., 2002). Given the known influence of rhythmic spike trains on synaptic efficacy (Bi and 


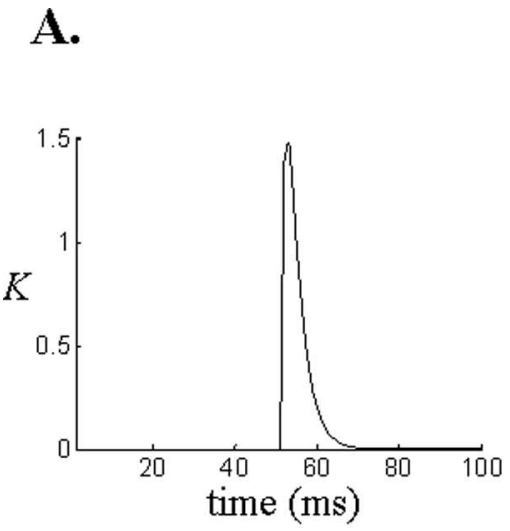

Figure 1. Functions for postsynaptic potential and long-term potentiation. $\boldsymbol{A}$, Temporal evolution of a postsynaptic potential when a spike is delivered at time $t=50$ (Eq. 2). $\tau_{\text {rise }}=1 \mathrm{~ms} ; \tau_{\text {fall }}=3 \mathrm{~ms}$. $B$, Change in synaptic efficacy $(\Delta w)$ as a function of the timing of presynaptic and postsynaptic spikes (Eq. 3). For parameters, see Table 1. [for more physiologically elaborate models, see, for example, Skinner et al. (2005) and references therein]. By default, the reversal potential is set much lower than the spike threshold to allow a slow ramping of activity. The membrane potential eventually reaches the spike threshold because of the addition of a small tonic current $\left(I_{\text {tonic }}\right)$. This tonic current is analogous to the addition of particular elements (e.g., potassium chloride) to in vitro preparations to promote spontaneous activity (Beggs and Plenz, 2003; Cossart et al., 2003).

Unless otherwise stated, synaptic efficacies $\left(\Delta w_{i j}\right)$ are adjusted according to STDP (Abbott and Nelson, 2000) as follows:

$\Delta w_{i j}(t)$

$$
=\left\{\begin{array}{cc}
W_{+} \cdot \exp \left(-\Delta t_{i j} / \tau_{+}\right) & \text {if } \Delta t_{i j}>0 \\
-W_{-} \cdot \exp \left(\Delta t_{i j} / \tau_{-}\right) & \text {if } \Delta t_{i j} \leq 0
\end{array}\right.
$$

Poo, 1999), neural responses to periodic stimuli may depend on changes in the interactions among cells.

To test this idea, we incorporated spike timing-dependent plasticity (STDP) in the model, enabling long-term changes in the strength of interactions between interconnected cells based on the timing of presynaptic and postsynaptic spikes (Abbott and Nelson, 2000). STDP has been found to promote neural synchronization in several neural systems and organisms. For instance, in the mushroom body of the locust, this mechanism helps preserve the propagation of odor-specific codes by enhancing neural synchronization (Cassenaer and Laurent, 2007). Here, we explore the idea that STDP may be able to encode the frequency of a train of stimuli delivered synchronously to a whole population of cells, ultimately providing insights into the neural mechanisms responsible for encoding temporal information.

\section{Materials and Methods}

Description of the model. Our starting point is an interconnected network of integrate-and-fire neurons. In this model, the membrane potential of individual cells tends naturally toward a resting potential; however, excitation received from neighboring cells can push the activity away from its resting state. The membrane potential $\left(V_{i}\right)$ of individual cells is updated as follows:

$$
\tau \frac{d V_{i}}{d t}=-g_{i}\left(V_{i}-E_{i}\right)+I_{\text {tonic }}+I_{i}+\sum_{j=1}^{N} w_{i j} K_{j},
$$

where cells are indexed from $i=1, \ldots, n, \tau$ is a time constant, $g_{i}$ is a leakage conductance, $E_{i}$ is a reversal potential, $I_{\text {tonic }}$ is a tonic current, $I_{i}$ is an external current (set to zero when purely spontaneous activity is considered), and $w_{i j}$ is a connection efficacy from cell $i$ to cell $j . K_{j}$ is the excitatory potential of incoming spikes (Gütig and Sompolinsky, 2006; Thivierge et al., 2007) described by the following:

$$
K_{j}=V_{0} \cdot \sum_{i=1}^{S} \exp \left(\frac{t_{i}-t}{\tau_{\text {fall }}}\right)-\exp \left(\frac{t_{i}-t}{\tau_{\text {rise }}}\right),
$$

where $t_{i}$ denotes the spike times of the $i$ th afferent and $V_{0}$ is a free parameter (Fig. 1A). Equation 2 computes the potential of incoming spikes based on a maximum of 10 previous spikes (indexed by $i=1, \ldots, S$ ). After the membrane potential of each cell is computed, a spike occurs if the spike threshold $V_{\text {thresh }}$ is exceeded. When a cell fires, its membrane potential is set to $V_{\text {spike }}$ for a time duration of $T_{\text {spike, }}$, after which it is reset to a resting potential of $V_{\text {rest }}$ and held there for a duration of $T_{\text {reset }}$. All membrane potentials are initialized to random values in the range $[0,1]$. Unless otherwise stated, the model only includes excitatory pathways where $\Delta t_{i j}=t_{i}-t_{j}$ reflects the difference between the last spike arrival times of presynaptic $\left(t_{i}\right)$ and postsynaptic $\left(t_{j}\right)$ cells. In STDP, increases in coupling strength are maximal when presynaptic pulses are immediately followed by a postsynaptic response; conversely, decreases in coupling strengths are maximal when presynaptic pulses are immediately preceded by postsynaptic activation (Fig. $1 B$ ). No hard bounding was applied to limit the range of synaptic weights, other than having to remain at positive values [i.e., by default, no inhibition was allowed in this simplified account, as in previous work (Nowotny et al., 2003)].

Weights are initialized to random values between $[0,10]$, with no self-connections. Weight updates are applied as follows: $w_{i j}(t)=w_{i j}(t-$ 1) $+\eta \Delta w_{i j}(t)$, where $\eta$ is a free parameter. There are four adjustable parameters in the STDP rule: $W_{+}$and $W_{-}$control the magnitude of change in synaptic efficacy; $\tau_{+}$and $\tau_{-}$control the time course of plasticity. Although a complete exploration of the parameter space of the model is beyond the scope of the current work, fine-tuning of the parameters was not necessary to achieve the results below (the effect of different parametric values is examined in Results). All simulations were performed using Matlab software, with an integration time step of $1 \mathrm{~ms}$.

Short-term adaptation. As an alternative to long-term changes in synaptic efficacy provided by the STDP rule (Eq. 3), we also consider a mechanism based on short-term adaptation. When a cell emits an action potential, several types of ionic channels become temporarily depleted, reducing the likelihood of another action potential for a particular time interval. For instance, fluctuations in intracellular calcium concentrations are strongly correlated with cortical synchronization (Ikegaya et al., 2004) and the likelihood of an UP state (i.e., a state of high activity) in cortex is greatly diminished when calcium concentrations are low. A simplified model was used to relate spike activity of a cell $i$ to the depletion and recovery of ionic resources over time as follows:

$$
U_{i}=-k \cdot \sum_{j=1}^{S} \exp \left(\frac{t_{j}-t}{\tau_{\text {fall }}^{\text {STA }}}\right)-\exp \left(\frac{t_{j}-t}{\tau_{\text {rise }}^{\text {SA }}}\right) .
$$

where $k$ is a parameter controlling the magnitude of depletion, whereas $\tau_{\text {fall }}^{\mathrm{STA}}$ and $\tau_{\text {rise }}^{\mathrm{STA}}$ control the time course of depletion and recovery, respectively. Synaptic efficacies are updated according to $\Delta w_{i j}(t)=\alpha U_{i}+\beta U_{j}$ $+\phi$, where $\alpha, \beta$, and $\phi$ are free parameters. Unless otherwise stated, all results use solely the STDP rule (Eq. 3), without short-term adaptation. However, Results (see below) also explores simulations that combine both short-term adaptation and STDP, as well as simulations with only short-term adaptation.

Initialization of parameters. Default parametric values used in simulations are provided in Table 1. To generate a heterogeneous population of cells, several of the free parameters of the model were not set to a single fixed value for all cells. Rather, a random Gaussian distribution of values 
Table 1. Default values of parameters and distributions

\begin{tabular}{ll}
\hline Parameters and distributions & Default values \\
\hline Spike parameters & $V_{\text {rest }}=1( \pm 0.5) \mathrm{mV} ; T_{\text {reset }}=3([1,8 \mathrm{~ms}]) \mathrm{ms} ; V_{\text {spike }}=100( \pm 10) \mathrm{mV} ; T_{\text {spike }}=1 \mathrm{~ms}$ \\
STDP parameters & $W_{+}=10( \pm 5) ; W_{-}=100( \pm 10) ; \tau_{-}=60( \pm 10) \mathrm{ms} ; \tau_{+}=30( \pm 10) \mathrm{ms}$ \\
Means of parameter distributions $(\sigma=0.33)$ & $\tau_{\text {fall }}=3( \pm 1) \mathrm{ms} ; E=-60( \pm 10) \mathrm{mV} ; \tau_{\text {rise }}=1( \pm 0.5) \mathrm{ms} ; g=0.01([-0.005,+0.05]) \mathrm{pS} ; V_{\text {thresh }}=18( \pm 3) \mathrm{mV}$ \\
Short-term adaptation parameters & $\tau_{\text {fall }}^{\text {STA }}$, mean of $200 ; \tau_{\text {rise' }}^{\text {SA }}$ mean of $2 ; k$, mean of $5 ; \alpha=0.5, \beta=0.5, \phi=10$ \\
Miscellaneous parameters & $I_{\text {tonic }}=1.1( \pm 0.5) \mathrm{mV} ; V_{0}=4( \pm 1) \mathrm{mV} ; \eta=0.5( \pm 0.2) ; \tau=0.01 ; n=100$ cells \\
\hline
\end{tabular}

The values in parentheses show a range of parameters that yield qualitatively similar results.

was defined independently for each of these parameters. Such a procedure has several advantages: (1) single-cell models are not constrained enough by data to justify precise values for some of these parameters; (2) even when experimental constraints are available, parametric distributions insure that the results obtained are robust to these values, and may thus generalize across cell types; (3) even within a small region of the brain, cells do not exhibit identical properties. Results of the heterogeneous model are compared with those of a homogeneous model in which parametric distributions are replaced by their means, therefore making all cells identical with one another.

Detecting network spikes. We define a NS as the synchronization of a large number of cells within a population, beyond the fortuitous coincidence of spikes that occurs by chance. To determine whether synchronized events constitute NSs, the following bootstrapping method is used. For each cell in the model, the following two steps are performed: (1) a binary vector of spike times is computed (with a $1 \mathrm{~ms}$ resolution), where " 0 " indicates that no spike occurred at that time, and " 1 " indicates that a spike was fired; (2) this spike vector is shuffled randomly.

After steps 1 and 2 are performed for all cells, two subsequent steps are performed: (3) the randomized spike times obtained in step 2 are grouped by summing across all spikes and all cells within individual time bins of $10 \mathrm{~ms}$. A bin size of $10 \mathrm{~ms}$ is standard practice in both experimental (Beggs and Plenz, 2003) and theoretical (Abbott and Rohrkemper, 2007) work. To insure that our main conclusions are not affected by this choice of bin size, we also performed simulations with an alternative bin size of $1 \mathrm{~ms}$. (4) A threshold is computed such that its value exceeds the summed activity from step 3 for at least $95 \%$ of the total recording time.

Steps 1-4 are repeated 1000 times, and a final threshold is computed by averaging over all values obtained in step 4 . This final threshold serves as the critical value to determine whether a NS is statistically reliable. To identify the precise timing of NSs, we apply this threshold to the original data (sampled at $1 \mathrm{~ms}$ ). A NS is detected when the sum of spikes exceeds the threshold (but does not exceed it at the previous time step). The maximal NS amplitude corresponds to the maximum number of concurrently active cells (i.e., within $1 \mathrm{~ms}$ ) during the time when the sum of spikes is above threshold.

Functional interactions. An important goal of the simulations presented here is to examine how changes in synaptic efficacy alter interactions among all cells in the population. From recent work, it is known that STDP can promote the formation of groups of neurons with strong mutual interactions (Izhikevich, 2006), but little is known about how these interactions change as a function of both spontaneous and induced activity. To investigate this issue, an algorithm termed Girvan-Newman clustering (Girvan and Newman, 2002) is applied to the matrix of synaptic connections before and after stimulation. The goal of this algorithm is to identify "communities" of neurons, characterized by strong withingroup interactions and weak between-group interactions. The algorithm achieves this by focusing on neurons that connect different communities; by removing these neurons, it reveals the underlying communities. For instance, the simple network of Figure 2 displays neurons according to their proximity (neurons with strong synaptic connections between them are close together in space). This network has three communities (shown by gray areas) that communicate with one another via a small subset of neurons (shown in red). These neurons have a high "betweenness centrality" (Freeman, 1977), referring to their importance in linking together the three communities; if these neurons are removed, the three communities become isolated.

The betweenness centrality of each neuron is defined as the fraction of

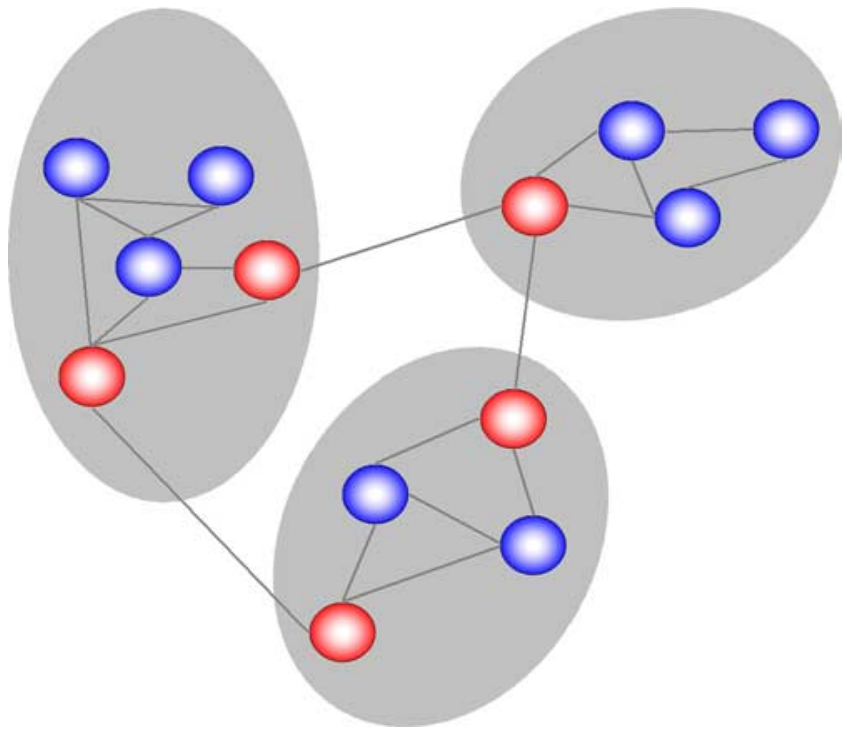

Figure 2. Neurons with high betweenness centrality play a fundamental role in the communication of different communities of cells. The gray areas show three communities. Neurons within each community are strongly interconnected but only weakly connected with other communities. Neurons linking communities together (shown in red) have a high betweenness centrality.

shortest paths between any pair of neurons that pass through that neuron. For a cell $i$, betweenness centrality is calculated as follows:

$$
B_{i}=\frac{1}{N(N-1)} \sum_{s \neq i \neq t} \frac{\sigma_{s t}(i)}{\sigma_{s t}},
$$

where $\sigma_{s t}(i)$ is the number of shortest paths (i.e., shortest number of intermediate cells) from neurons $s$ to $t$ that pass through neuron $i$, and $\sigma_{s t}$ is the number of all shortest paths linking $s$ to $t$. Matlab code used to calculate this algorithm is available online (Gleich, 2006). Neurons that connect different communities will have a high betweenness centrality, because they will often provide the shortest path between members of different communities.

Second, the neuron with highest betweenness centrality is removed. The algorithm then begins from step 1 again, recalculating betweenness centrality, and iterating until no neurons remain. The result is a tree of community memberships. To identify particular communities from this tree, we first set a cutoff point for the maximum number of communities to consider, and then label neurons along the same branch, as done previously (Girvan and Newman, 2002).

\section{Results}

\section{Nonperiodic synchronization}

In a population of cells simulated for $5 \mathrm{~min}$, spontaneous activity arises in a highly nonperiodic manner, forming several of NSs with strong statistical reliability (Fig. $3 A$ ). As in experimental recordings of rat cortex (Eytan and Marom, 2006), the temporal interval separating different NSs is not constant; rather, there is a distribution of intervals ranging from $\sim 1$ to $>5 \mathrm{~s}$ (Fig. $3 B$ ). 
A.
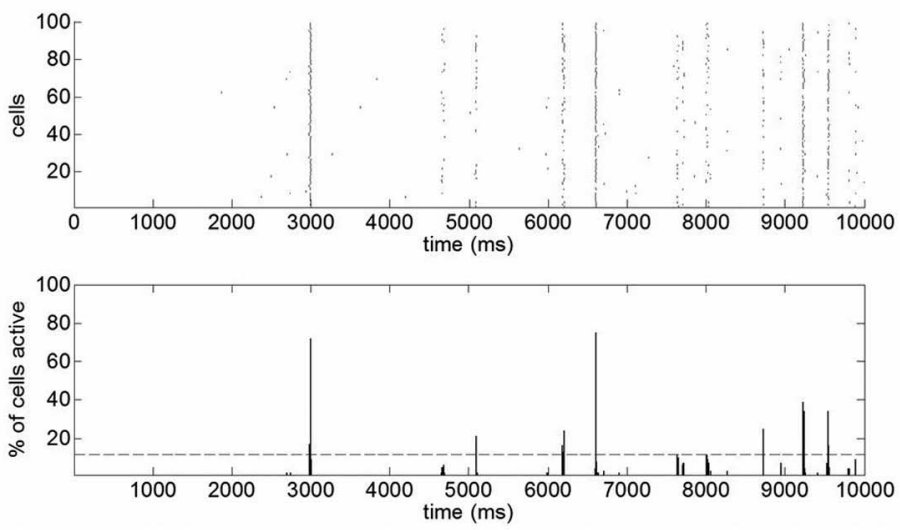

B.

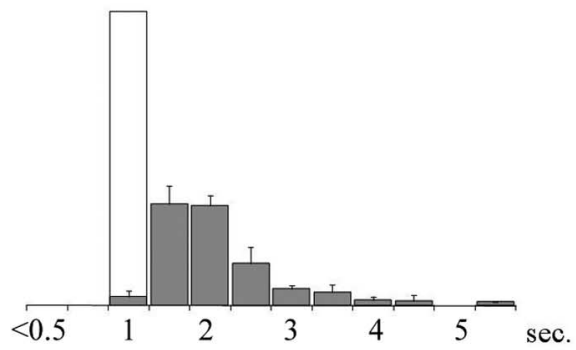

C.

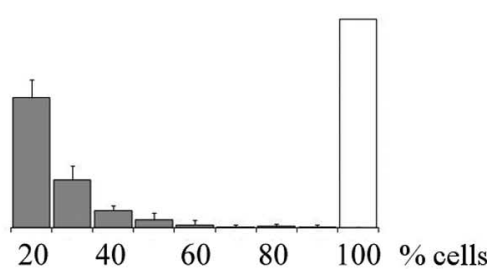

Figure 3. Nonperiodic network spikes. A, Example of a 10 s simulation. Top, Rasters indicate the firing times of individual cells in a population of 100. Bottom, Percentage of cells active in nonoverlapping $10 \mathrm{~ms}$ bins. The dashed line shows statistical significance of activity at $p<0.05$ (obtained through bootstrap reshuffling). $\boldsymbol{B}$, Distribution of intervals between statistically reliable NSs. The vertical bars show SEM for 10 independent runs of the model. Gray bars, Heterogeneous model. White bar, Homogeneous model. C, Distribution of the percentage of active cells during NSs.

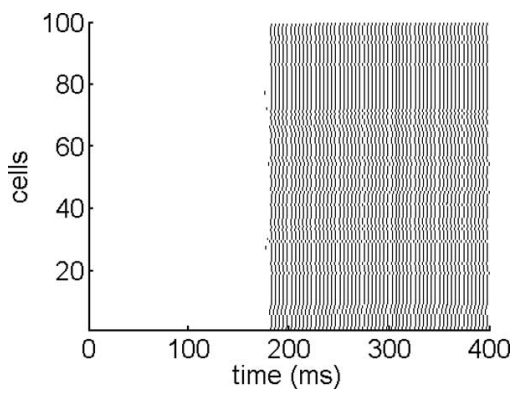

Figure 4. Simulation of spontaneous activity without STDP. Without STDP, the positive feedback between neurons causes persistent firing which is never aborted.

Because the above simulation did not include any stochastic fluctuations in the membrane potential, the nonperiodicity of NSs cannot be accounted for by such random noise. Rather, nonperiodicity is a direct consequence of cellular heterogeneity: when the latter is completely eliminated in a simulation in which all cells have identical properties, synchronization is exactly periodic (generating NSs at $1 \mathrm{~Hz}$ ) (Fig. 3B, white bar).

In addition to accounting for the nonperiodic intervals between NSs, cellular heterogeneity is also responsible for generating a relatively broad spectrum of peak amplitudes in the number of cells active across different NSs (Fig. 3C), as found experimentally (Eytan and Marom, 2006). By comparison, a homogeneous simulation always recruits all cells during NSs.

Although cellular heterogeneity represents a potential means of generating nonperiodic synchronization, it is insufficient by itself to explain why synchronization arises. In this respect, plasticity plays an important role. The role of STDP in neural synchronization is highlighted in a simulation in which connections remain fixed at their initial values. In this case, synchronized activity still occurs, but is never terminated (Fig. 4). Once a NS is initialized by positive feedback in the interaction among excitatory cells, there is no longer any means of stopping it, and high-frequency synchronized activity is perpetuated until the simulation is stopped.

Together, the above results argue that a basic mechanism combining STDP and cellular heterogeneity can account for the generation of NSs with broad distributions of time intervals and peak amplitudes, as reported experimentally (Eytan and Marom, 2006). Note that, although the heterogeneous model did not include stochastic fluctuations in membrane potential, the addition of noise does not preclude the formation of NSs (supplemental Table 1, available at www.jneurosci.org as supplemental material). In biological networks, nonperiodic synchronization may therefore reflect a combination of factors, including cellular heterogeneity and stochastic fluctuations in membrane potential.

How much does nonperiodic synchronization depend on the various parameters used in the model? We addressed this question by examining the effect of varying all of the parameters of the model (Table 1) and plotting the resulting distribution of time intervals between NSs for a 5 min simulation of spontaneous activity (supplemental Table 1, available at www.jneurosci.org as supplemental material). As depicted, most of the parameters can be altered and still yield a broad distribution of inter-NS intervals.

In one variant of the model, we introduced a distribution of delays in synaptic transmission in the range $[1,20] \mathrm{ms}$ across the different cells of the population (supplemental Table 1, "with delays," available at www.jneurosci.org as supplemental material). Even when these delays form the only source of heterogeneity (all other parametric distributions collapsed onto their means), a broad distribution of NS intervals emerges, in agreement with previous work (Izhikevich, 2006).

In another variant of the model, we reduced the density of connections between cells by permanently removing a percentage of randomly chosen connections at the beginning of the simulation. In this way, up to $20 \%$ of connections could be removed without eliminating the occurrence of NSs. A similar result is found when permanently replacing a percentage of randomly chosen connections with inhibitory values $\left(w_{i j}=-1\right)$; the network can withstand the addition of up to $30 \%$ of inhibitory pathways and still produce nonperiodic NSs. Interestingly, changes to the network parameters sometimes have an unpredictable impact on network dynamics. For instance, the addition of $30 \%$ of inhibition increases the proportion of NSs with $<500 \mathrm{~ms}$ of temporal distance. 
By adjusting the reversal potential and mean resting potential of the model, one variant of the model approximates values reported for cortical neurons $\left(E=V_{\text {rest }}=\right.$ $-65 \mathrm{mV}$ ) (Dayan and Abbott, 2001). In this variant, two parameters of central importance are the tonic current $\left(I_{\text {tonic }}\right)$ and mean spike threshold $\left(V_{\text {thresh }}\right)$. An increased tonic current reduces the distance between NSs by increasing the slope of the rising membrane potential, whereas a decreased spike threshold achieves a similar effect by making individual cells fire when a lower potential is reached (Fig. 5).

In a final variant of the model, we replace the STDP rule with a short-term adaptation rule representing the temporary depletion of ionic resources after an action potential (Eq. 4). This rule enables the production of NSs that are stopped when ionic resources are depleted (Fig. 6A). Short-term adaptation can also be combined with STDP, resulting in a model with both faster and slower changes in synaptic efficacy. In such a model, it is possible to impose STDP modifications that cannot occur faster than every $10 \mathrm{~s}$, and still obtain NSs (albeit with a narrower distribution) (compare Fig. $3 B$ ). Although previous modeling efforts have forced STDP updates every $1 \mathrm{~s}$ (Izhikevich, 2006), we show that longer, more realistic time courses also yield NSs if short-term adaptation is part of the model. Next, we demonstrate how the combination of STDP and heterogeneity can account for other important aspects of NSs.

\section{Exponential growth in activity}

A key property of cortical NSs is the rate at which more and more cells become active up to the peak of a NS, fitting an exponential population growth function (Eytan and Marom, 2006). This effect is captured in simulations by first storing the spontaneous activity of all cells for $60 \mathrm{~s}$. Then, all NSs are aligned in time according to their peak activity (Fig. 7A). Taking the mean spike rate across all peaks and all cells, we observe an exponential increase in activity up to the peak of this "grand average NS" (Fig. $7 B)$. As in experimental work, we fit this grand average NS with the following function: $A(t)=a+b \cdot e^{(\sigma-1) \cdot t}$. The parameters $a, b$, and $\sigma$ were adjusted to obtain the best possible fit by minimizing the sum of squared errors between the data points and function. Although the model was in no way optimized to match experimental results, the fitted values for the parameters of the exponential function are relatively close to values previously reported for rat cortical slices: $a=0.012$ (cortex, 0.05$), b=0.011$ (cortex, 0.01), and $\sigma=1.106$ (cortex, 1.045) (Eytan and Marom, 2006). This result arises with no explicit rule or mechanism for enabling the exponential recruitment of cell activity; rather, it is a property that emerges simply through positive feedback in an interconnected network. In addition, the temporal precision of the grand average NS obtained in the model offers a close match to that observed in cortex, with a total duration (beginning with the initial rise of activity and ending with its return to baseline levels) of $\sim 100 \mathrm{~ms}$.

In recent in vitro experiments (Eytan and Marom, 2006), NSs recruit the greater majority of electrodes in a cortical preparation.
B.

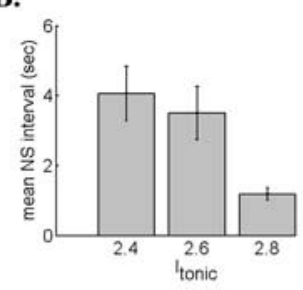

D.

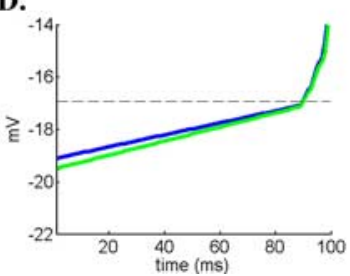

C.
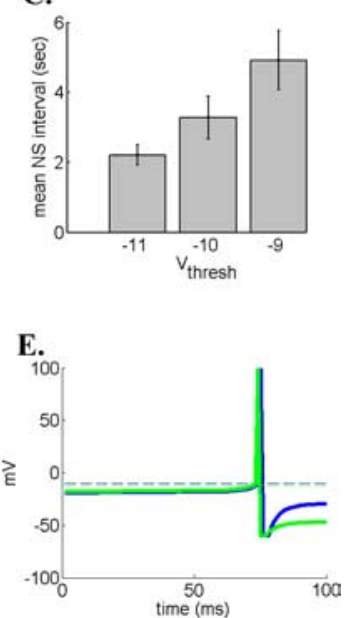

Figure 5. Influence of tonic current and mean spike threshold on temporal intervals between NSs. $\boldsymbol{A}$, Distribution of time intervals between NSs for a version of the model with reversal potential and resting potential approximating those of cortical cells $V_{\text {rest }}=-65 \mathrm{mV}$ ). $I_{\text {tonic }}=2.6 \mathrm{mV} ; V_{\text {thresh }}=-10 \mathrm{mV} . \boldsymbol{B}, \boldsymbol{C}$, Increasing tonic current or decreasing spike threshold reduces are identical except for their tonic current. The dashed line indicates spike threshold. $\boldsymbol{E}$, Increasing the spike threshold delays the

A similar result emerges in simulations: when summing the total number of active cells over a $200 \mathrm{~ms}$ window surrounding each NS (from -100 to +100 ms with respect to peak activity), we find that a large proportion of NSs recruit $\sim 80 \%$ and more of cells; in fact, over a 5 min simulation, only a small minority of NSs recruits $<50 \%$ of cells. Synchronization can thus be considered a relatively global phenomenon within a population of cells, as opposed to smaller, more local interactions among small clusters of cells (see Fig. 13) (discussed below).

\section{A subpopulation of cells is highly sensitive to the imminence of a network spike}

In vitro recordings have suggested the presence of cells that preferentially increase their activity immediately before NSs (Eytan and Marom, 2006). To investigate this phenomenon in the model, we first performed a within-cell regression relating the correlation between firing rates before NSs (100 ms before peak) and firing rates at the peak of NSs. This analysis identified a subgroup of cells ( $57 \%$ of the population) that reliably increased their activity before NSs $(p<0.01 / N$, Bonferroni-corrected for each cell in the network). Following recent experimental results, we refer to this subgroup as "early-to-fire" cells (Eytan and Marom, 2006).

To more clearly segregate cells in the network according to their firing rates before NSs, we followed the above analysis with a between-cell regression examining the mean firing rates of early-to-fire cells versus other cells in the model. Across early-tofire cells, an increased firing rate before NSs was predictive of an increased firing rate at the peak of NSs (Fig. 8, cells marked in blue) (linear regression over all these cells: $r^{2}=0.40, p<0.002$, after removing a single outlier cell with firing rate $>4$ SDs above the mean). Across the remaining cells, in which no statistically reliable relationship could be established between activity before and during NSs, a trend was observed in the opposite direction: higher firing rates before NSs were linked to lower firing rates 
A.
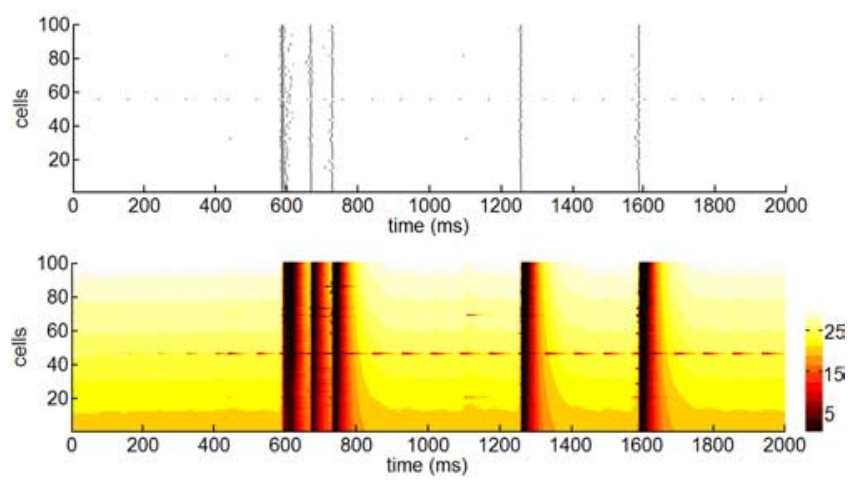

B.
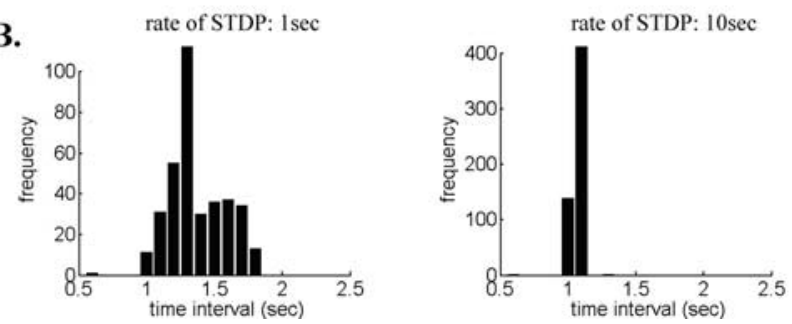

Figure 6. Short-term adaptation gives rise to network spikes. $\boldsymbol{A}$, Top, Rasters indicate the firing times of individual cells across time. Bottom, Changes in $w_{i j}$ (short-term depletion of ionic resources) after NSs (Eq. 4). B, Distribution of time intervals between spontaneous NSs for simulations with a Gaussian distribution of time delays for updates to synaptic changes (centered at either 1 or $10 \mathrm{~s}$, with SD $\sigma=0.33$ ).

during NSs (Fig. 8, cells marked in red) (linear regression over all these cells: $\left.r^{2}=-0.77, p<0.001\right)$.

Hence, the heterogeneous population cells in the model divided itself into two subgroups. For one subgroup, increased activity before NSs was linked to decreased activity during NSs; for the other subgroup, a similar increase in activity before NSs was linked to an increase in activity during NSs. The latter subgroup represents a subset of cells whose activity is highly predictive of the imminence of NSs, as found in cortical preparations (Eytan and Marom, 2006).

\section{Neural stimulation leads to an all-or-none response}

The above simulations on the exponential growth of cell activity preceding NSs demonstrate that, despite a great deal of unpredictability in the time interval between NSs, some aspects of spontaneous activity display a high degree of predictability. A similar conclusion is reached when simulated cells are stimulated by external currents of different intensities (Fig. 9A,B). As in cortical networks (Eytan and Marom, 2006), population activity responds to stimulation in an all-or-none manner: when the stimulation intensity is above a certain threshold of intensity ( $\sim 600 \mathrm{mV}$, in which all cells are stimulated for $5 \mathrm{~ms}$ ), the majority of cells are activated (i.e., the population "ignites"). Below this threshold of intensity, the population of cells remains at a low baseline of activity.

A comparable phenomenon of threshold-governed population response is observed when the number of simultaneously stimulated cells is varied (Fig. $9 C-E$ ). When less than $\sim 10 \%$ of cells are stimulated concurrently ( $1000 \mathrm{mV}$ current, lasting $5 \mathrm{~ms}$ ), the induced activity remains localized to a relatively small number of cells. When $10 \%$ or more of cells are stimulated in the same manner, the induced activity spreads to a large proportion of cells in the population.
The above results suggest the presence of a "critical point" in the number of cells stimulated and the intensity of stimulation, beyond which there is activation of a majority of cells in the population. In the model, the presence of this critical point is strictly implicit and was never directly enforced by the design of the simulations. Of course, the particular value of this critical point depends on the choice of model and parameters, which could be adjusted to fit particular experimental constraints; here, we offer a proof-of-principle that a model based on simple neural interactions can capture the "ignition" effect observed experimentally (Jimbo et al., 1999; Eytan et al., 2003; Eytan and Marom, 2006).

\section{Rhythmic stimulation generates an echoic trace}

The ability of simulated cells to respond in a reliable manner after stimulation (i.e., after an all-or-none NS) suggests that, although some aspects of spontaneous activity are seemingly unpredictable, the network nonetheless responds in a systematic and predictable way to external stimulation. To explore this possibility further, we aimed to determine whether the response of the network could be influenced by the temporal structure of a sequence of stimuli (Yoshioka, 2002). There is a substantial body of experimental literature supporting this idea: for instance, Mikkonen et al. (2002) examined the response of compound action potentials after tetanization at different frequencies. Between $\sim 30$ and 50 $\mathrm{Hz}$, the frequency of occurrence of compound action potentials after tetanization mimicked that of the stimulation. In a similar vein, the termination of rhythmic evoked potentials elicits a neural response at the approximate time at which the next stimulus would have occurred. This phenomenon, termed "omitted stimulus potential," is found across several species, from humans to honeybees and ants, and forms the neural substrate of a basic form of cognitive expectation (Ramón and Gronenberg, 2005).

To examine whether our model reproduces this phenomenon, the following simulations were performed. First, spontaneous activity was recorded for $60 \mathrm{~s}$ (this constitutes the baseline epoch). Next, an external stimulation was applied at a fixed frequency (e.g., $10 \mathrm{~Hz}$ ) (Fig. 10A) (current intensity, $1000 \mathrm{mV}$; pulse duration, $5 \mathrm{~ms}$ ) for a duration of $60 \mathrm{~s}$. Finally, the stimulation was terminated and spontaneous activity again recorded for $2 \mathrm{~s}$. During the baseline epoch, NSs occurred in a nonperiodic manner and with low frequency (Fig. $3 A, B$ ), as discussed previously. When a current was applied, NSs were generated at a mean frequency close to that of the stimulation (Fig. $10 \mathrm{~B}$, particularly between 1 and $50 \mathrm{~Hz}$ ). Finally, when stimulation was terminated, the mean frequency of NSs did not immediately revert to baseline levels. Rather, this frequency "echoes" that of the stimulation for a few cycles ( $<1000 \mathrm{~ms}$ poststimulation). This behavior of the model, which we term an "echoic trace," was observed over a range of frequencies from $\sim 1$ to $50 \mathrm{~Hz}$ (Fig. $10 \mathrm{~B}$ ). It represents a transient state of synchronization in which spontaneous NSs can occur at a much higher rate than observed at prestimulation baseline, in a way that resembles the induced frequency of stimulation. A theoretical treatment of the role of STDP in sequence learning is beyond the scope of the current work and can be found in previous studies (Suri and Sejnowski, 2002; Yoshioka, 2002; Chao and Chen, 2005; Aoki and Aoyagi, 2007; Jun and Jin, 2007; Masuda and Kori, 2007; Hosaka et al., 2008; Kube et al., 2008; Masquelier et al., 2008;). Consistent with this work, our results argue that STDP may be a key mechanism for learning temporal sequences; indeed, a simulation that removes STDP but retains short-term adaptation does not exhibit an echoic trace that mimics the frequency of induced stimulation (Fig. 11). 
Despite the short lifetime of an echoic trace, neural stimulation has long-term effects on the spike dynamics of the model. This point is illustrated by examining the spiking activity of three independent simulations with no stochastic fluctuations in membrane potential, and identical initial states (Fig. 12). For the first $1100 \mathrm{~ms}$ after initialization, the activity of these three simulations was identical. Then, a single pulse of stimulation was delivered with only $1 \mathrm{~ms}$ difference between the three simulations (i.e., at $1100 \mathrm{~ms}$ for the first simulation, $1101 \mathrm{~ms}$ for the second simulation, and $1102 \mathrm{~ms}$ for the third simulation). As a result of this subtle time difference, the spiking activity of the three simulations diverged noticeably, with repercussions well beyond the time of stimulation [in fact, differences across conditions can be observed for as long as the simulation was carried (i.e., $2000 \mathrm{~ms}$ )]. These simulations argue for the idea that, even in purely deterministic cellular networks, spike activity can be influenced by a long preceding history.

\section{Population-wide synaptic interactions}

Are the interactions among cells altered as a function of neural stimulation? If so, what is the relationship between synaptic modification and the ability of cells to echo at certain frequencies? To address these questions, we applied the Girvan-Newman clustering algorithm to the matrix of synaptic connections immediately before and after $10 \mathrm{~Hz}$ stimulation (this frequency was chosen because it yields a strong echoic trace) (Fig. 10A). Before stimulation, a large community of cells emerges among the network of interactions, accompanied by a number of other markedly smaller communities (Fig. 13A, left). To facilitate visualization, Figure 13 displays the network of neurons according to a multidimensional scaling (MDS) (Davison, 1983). MDS finds a set of coordinates in two-dimensional space such that the Euclidean distances among neurons corresponds as closely as possible to their shortest paths (i.e., shortest number of intermediate cells) (Sporns, 2002). The result is a graph that shows how closely two neurons are interacting, with some neurons communicating directly with one another (i.e., short path lengths) and others communicating only through other neurons (i.e., longer path lengths).

For every cell in the network, we computed a cross-correlation function and compiled these results in a histogram of preferred (strongest) correlations for each community (Fig. 13B). To control for spurious correlations, we performed a 100 -fold bootstrap reshuffling of the spike times of each cell. Any portion of the cross-correlation function was considered statistically reliable if it exceeded at least $99 \%$ of the shuffled cross-correlations. Before stimulation, the histogram of the largest community reveals a strong preferred correlation within $\pm 10 \mathrm{~ms}$ of time lag, arguing for the absence of long temporal dependencies between the activity of different pairs of cells (Fig. 13B, top left). A similar profile is found for all three of the largest communities of the prestimulation network.

After stimulation, synaptic interactions are markedly altered, and the network breaks apart into three distinct communities (Fig. 13A,

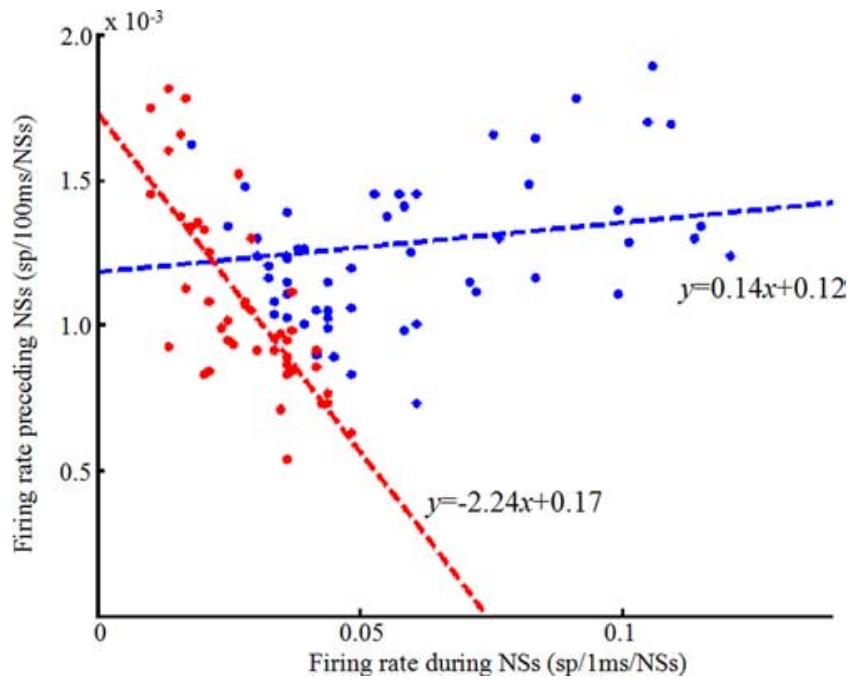

Figure 8. Relationship between mean firing rates before NSs and during NSs. The scatter plot shows cells with either a significant (blue dots) or nonsignificant (red dots) correlation between activity before and during NSs. The dashed lines are corresponding linear regressions for the activity of these cells. Firing rates are means over both NSs and time window (during NSs, $100 \mathrm{~ms}$; at peak of NSs, $1 \mathrm{~ms})$. sp, Spike.

middle). As a result, the network of interactions becomes strongly modular, combining multiple within-community ties with few between-community ties. In addition, the largest community of the poststimulation network (Fig. 13, blue cells) has a crosscorrelation histogram that differs in a meaningful way from that of the largest prestimulation community: in addition to a peak at $0 \mathrm{~ms}$ time lag, a smaller peak is visible at $-100 \mathrm{~ms}$, as well as small peaks near $+90 \mathrm{~ms}$ time lag. These peaks occur above chance (when compared with the shuffled cross-correlograms) and are 
A

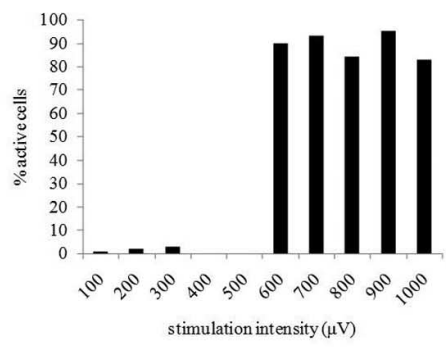

C.

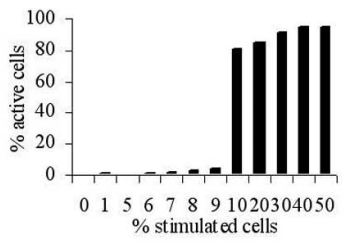

B.

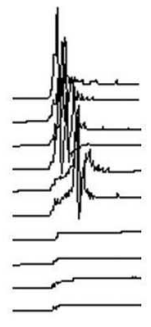

$5 \mathrm{sp} / \mathrm{ms} / 100$ cells

$20 \mathrm{~ms}$
E.

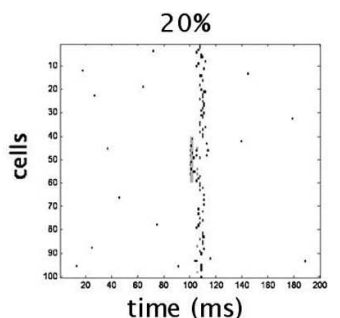

Figure 9. All-or-none population response to stimulation. $A$, Percentage of cells active in response to different intensities of stimulation. $\boldsymbol{B}$, Sum of spikes across all cells given different stimulation intensities, ranging from $100 \mathrm{mV}$ (bottom trace) to 1000 $\mathrm{mV}$ (top trace). $\boldsymbol{C}$, Percentage of cells active given different percentages of cells stimulated. $\boldsymbol{D}, \boldsymbol{E}$, Examples of population activity when either 5 or $20 \%$ of cells are stimulated (stimulated area shown in gray). Stimulation was performed at $1000 \mathrm{mV}$ for a duration of $1 \mathrm{~ms}$. ( $A$, $C$, Spikes were summed across bins of $10 \mathrm{~ms}$.) sp, Spike.

A.

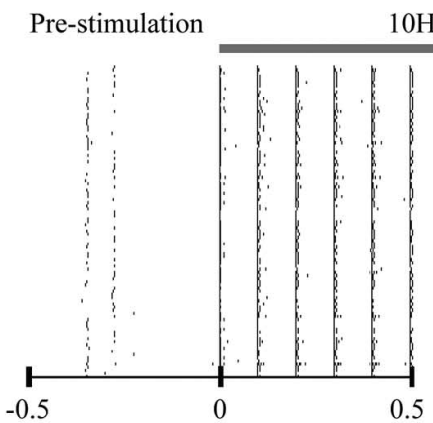

$10 \mathrm{~Hz}$ Stimulation

Post-stimulation
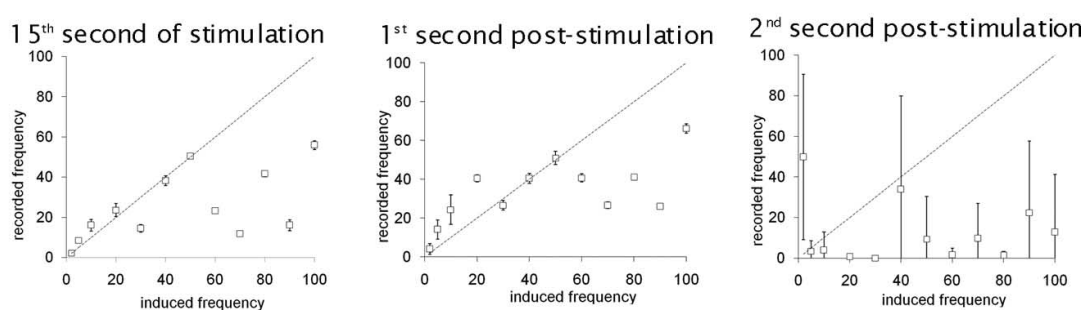

Figure 10. Echoic trace effect. $\boldsymbol{A}$, Spiking activity of simulation across all cells. The left window shows activity for the last $0.5 \mathrm{~s}$ before onset of stimulation and the first $0.5 \mathrm{~s}$ of activity after onset of stimulation (the $x$-axis is time in seconds relative to onset of stimulation). The right window shows spiking activity for the last $0.5 \mathrm{~s}$ of stimulation and the first $0.5 \mathrm{~s}$ of activity after stimulation is terminated. The stimulation was delivered to all cells simultaneously, at a frequency of $10 \mathrm{~Hz}$ (gray overbar shows the window of stimulation). $\boldsymbol{B}$, Frequency of NSs for different time windows (averaged over 10 independent runs), in response to different frequencies of induced stimulation. The dashed line corresponds to unity. The vertical bars are SEM.

consistent with an echoic trace at $10 \mathrm{~Hz}$ frequency. They are only present in the largest community of cells; the second and third largest communities do not exhibit such peaks. These results suggest that the echoic trace effect can be localized within a network of interacting cells; although a large group of cells contributes to this effect, others do not.

The presence of distinct communities in the poststimulation network likely depends on precise synaptic interactions, because they cannot be discriminated by firing rates alone (Student's $t$ test, red vs green communities, $t_{(51)}=2.01, p>$ 0.074 ; blue vs red, $t_{(67)}=2.00, p>0.779$; blue vs green, $\left.t_{(58)}=2.00, p>0.430\right)$; similar analyses attempting to discriminate among communities based on cell properties (see parameters of Table 1) or initial synaptic weights (i.e., average weights either sent or received by each cell) also fail to reach statistical significance ( $p>0.05)$. Finally, the fragmentation of the functional network into distinct modules is a short-lived effect. After the third second poststimulation, the network returns to a single large community from which only a few cells are excluded (Fig. 13A, right).

\section{Discussion}

Highly synchronized, nonperiodic neural activity is a widely reported phenomenon in spontaneously active populations of cells (Cossart et al., 2003; Eytan and Marom, 2006). The simulation results provided here argue that network spikes may result from positive excitatory feedback originating from a recurrent connectivity between cells. When such feedback gets strong enough, all cells in the population fire in close temporal contiguity. At that point, a large proportion of cells fall in a refractory state that prevents the immediate generation of additional action potentials, causing the NS to terminate. As a result, activity will desynchronize. In the model, the nonperiodic occurrence of NSs is a direct consequence of heterogeneous cellular properties. In a homogeneous simulation in which all cells have the exact same intrinsic properties, synchronization becomes strictly periodic, and individual NSs always recruit the whole population of cells. In the model presented here, no additional stochasticity (beyond the random initialization of cell properties) is required to produce a relatively broad distribution time intervals between NSs. This suggests that the nonperiodic synchronization observed in cortical networks may be more strongly dependent on the network-wide interactions of a heterogeneous population of cells than on small stochastic fluctuations in the membrane potentials of individual neurons.

A direct prediction from our simulation results is that the downregulation of endogenous or activity-driven factors responsible for cellular heterogeneity would result in a more periodic synchronization of cell activity. For example, BDNF upregulation has been argued to 
shift the distribution of synaptic conductances upward in a population of cells, leading to a skewed distribution and increased cellular homogeneity, with (as predicted by our simulations) marked increase in the periodicity of NSs (Fujisawa et al., 2004).

Although spontaneous NSs do not follow a precise period of occurrence, the model can be entrained to produce an echoic trace, that is, NSs that approximate the period of induced stimulation. The adjustment of synaptic strengths through STDP plays a crucial role in the formation of this echoic trace. When this property is removed from the model, or replaced with short-term adaptation, spontaneous NSs are preserved, but the poststimulation activity no longer approximates the induced stimulation.

After stimulation, STDP induces a marked reorganization of the functional interactions among connected cells, leading to a fragmentation of cells into communities with distinct crosscorrelation functions. The observed fragmentation of functional interactions is unlikely to be caused by some bias in the initial state of the system before simulation. Indeed, neither did intrinsic cell parameters nor synaptic strengths correlate reliably with community membership. Rather, communities are formed as a result of spike interactions that are reinforced through time by synaptic plasticity. A logical extension of this conclusion is that it might be possible to slow down the return of synaptic interactions to their prestimulation state by inhibiting spontaneous activity immediately after stimulation. Because of the complexity of spike interactions, and the effect that millisecond differences in spike timing have on overall dynamics (Fig. 12), predicting the exact number of communities in the poststimulation network of interactions as well as which particular cells will fall into one community or another would likely require an analysis whose complexity approaches that of the system under study.

Although other modeling approaches have examined the role of STDP under different forms of neural stimulation (Tsuda, 2001; Suri and Sejnowski, 2002; Yoshioka, 2002; Nowotny et al., 2003) as well as spontaneous activity (Izhikevich, 2006), the current work shows how a single model can account for established experimental findings relative to both spontaneous and induced NSs. When simulated NSs are induced with a rhythmic stimulation, the model demonstrates the ability to transition from a state of spontaneous, nonperiodic synchronization, to a stimulusinduced, more periodic state of activity. Hence, the tendency of the model to spontaneously produce nonperiodic NSs does not preclude the generation of more ordered responses under stimulation (Marom and Shahaf, 2002).

Given the ubiquity of spontaneous activity across species and brain regions (Raichle, 2006), could it have a functional relevance? One suggestion is that nonperiodic forms of spontaneous activity may facilitate responses to external stimuli by preventing resting-state activity from getting permanently "stuck" in a particular state (i.e., a so-called attractor state). We thus conjecture that the upshot of nonperiodic forms of activity may be to enable a great deal of flexibility and rapid responsiveness to external events. In future work, this conjecture could be extended further by using the model proposed here to encode more behaviorally and cognitively relevant stimuli. Studies aimed at this goal are already beginning to emerge. For instance, a recent theoretical
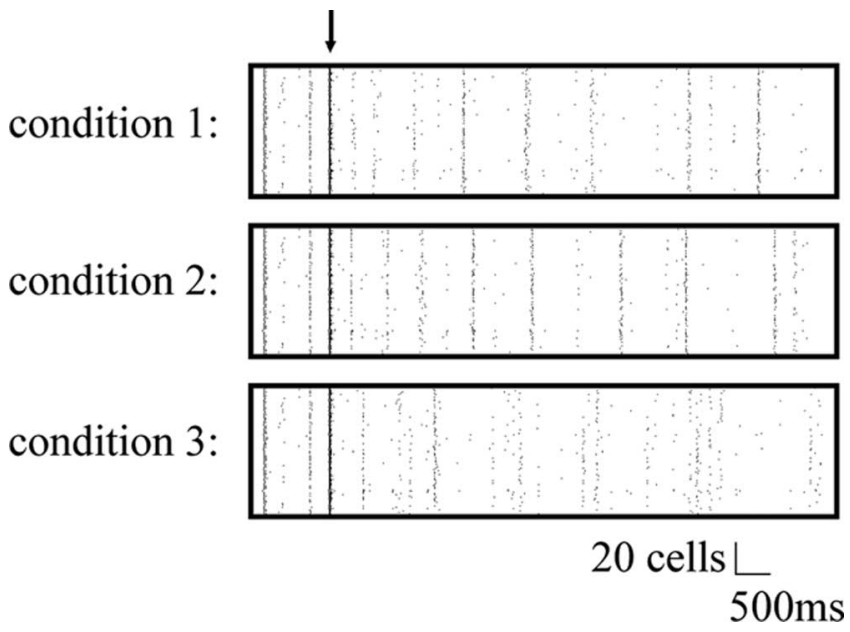

Figure 12. Millisecond precision of spike dynamics in the model. Three different simulations each run with identical initial conditions. The top condition receives a single pulse of stimulation at time $1100 \mathrm{~ms}$ (approximate temporal location indicated by the arrow above the top raster), and the subsequent conditions receive the same pulse 1 ms later (i.e., at 1101 and $1102 \mathrm{~ms}$ ). Pulses were delivered at $1000 \mathrm{mV}$ and lasted for $5 \mathrm{~ms}$. Despite the stimulation being delivered extremely closely in time across the three conditions, notice the marked divergence in spike dynamics, lasting well beyond the time of stimulation.

model based on STDP (Koene and Hasselmo, 2008) captures the replay of spatiotemporal sequences of spikes observed in hippocampus during slow-wave sleep (Lee and Wilson, 2002) as well as consummatory behavior (Foster and Wilson, 2006). More generally, the well documented role of STDP in learning temporal sequences (Abbott and Blum, 1996; Bi and Poo, 1999) makes it a promising avenue of exploration toward uncovering the basic mechanisms of learning and memory.

The current work is a step forward in bridging spontaneous and stimulus-driven forms of synchronization, and the model developed offers a relatively good match to some aspects of experimental data (for instance, in fitting an exponential function to the growth of population activity preceding NSs). However, we do not provide a comprehensive coverage of the extensive literature on the statistics of spontaneous and evoked activity. Additional work will be required to capture other specific findings, including (but not limited to) neural avalanches (Beggs and Plenz, 2003) and so-called "cortical song" (Ikegaya et al., 2004), both constituting highly nonrandom forms of spontaneous activity. Recent modeling work is moving in that direction (Abbott and Rohrkemper, 2007).

In conclusion, although the proposed model is a simplified account that remains to be enriched by additional biophysical 
A.

Pre-stimulation

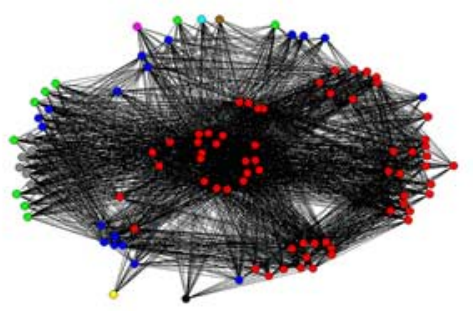

B.
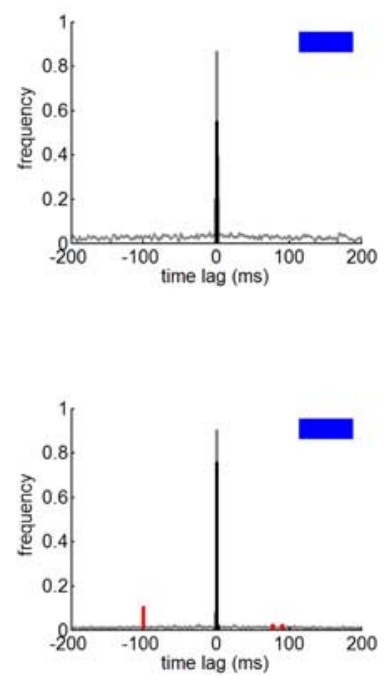

Post-stimulation (1st sec)

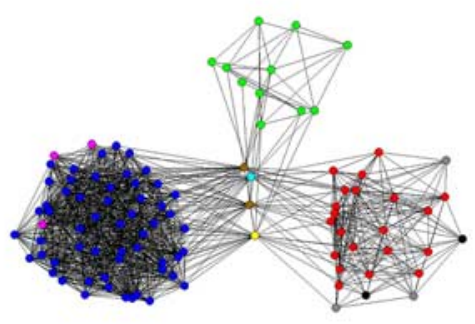

Pre-stimulation

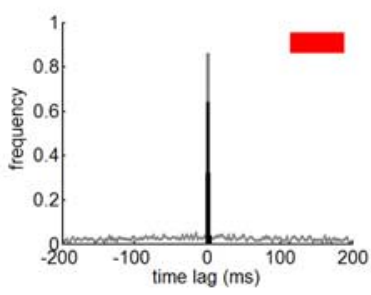

Post-stimulation (1st sec)

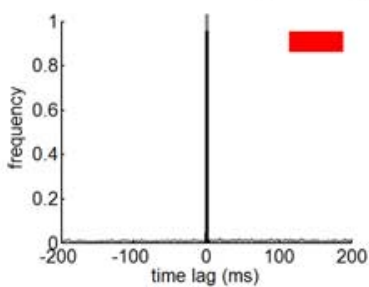

Post-stimulation (3rd sec)

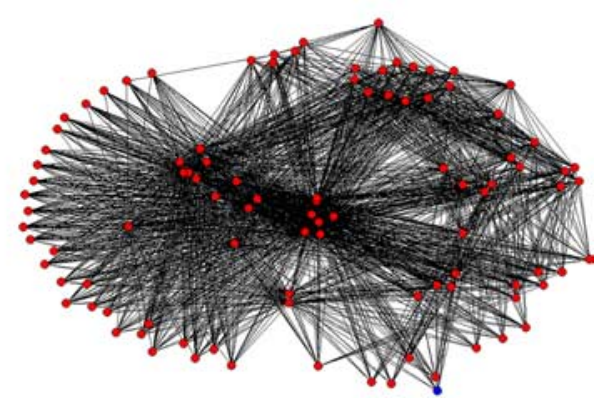

Figure 13. The network of synaptic interactions among cells is restructured after a rhythmic $10 \mathrm{~Hz}$ stimulation. $A$, Girvan-Newman analysis of synaptic connectivity before and after stimulation. Different communities are labeled by color for each cell in the network (for ease of visualization, only the top 10 largest communities are colored). Neurons are arranged in space according to a multidimensional scaling with high goodness-of-fit (stress values, $<0.2)$. B, Population cross-correlograms for the three largest communities in the prestimulation and poststimulation networks (corresponding community shown by color in top right of each figure). Gray lines, Average cross-correlogram obtained by 100 -fold bootstrap reshuffling of the spike times. Temporal locations at which the population cross-correlogram crosses at least $99 \%$ of the shuffled cross-correlograms are shown in red.

details, additional developments will likely not overturn the fundamental property of this simple account to produce nonperiodic synchronization under reasonable conditions. The nonperiodic synchronization of simulated cells may represent a natural and unavoidable consequence of spontaneous cell interactions in numerous regions of the brain.

\section{References}

Abbott LF, Blum KI (1996) Functional significance of long-term potentiation for sequence learning and prediction. Cereb Cortex 6:406-416.

Abbott LF, Nelson SB (2000) Synaptic plasticity: taming the beast. Nat Neurosci 3 [Suppl]:1178-1183.

Abbott LF, Rohrkemper R (2007) A simple growth model constructs critical avalanche networks. Prog Brain Res 165:13-19.

Aoki T, Aoyagi T (2007) Synchrony-induced switching behavior of spike pattern attractors created by spike-timing-dependent plasticity. Neural Comput 19:2720-2738.

Axmacher N, Mormann F, Fernández G, Elger CE, Fell J (2006) Memory formation by neuronal synchronization. Brain Res Rev 52:170-182.

Beggs JM, Plenz D (2003) Neuronal avalanches in neocortical circuits. J Neurosci 23:11167-11177.

Bi G, Poo M (1999) Distributed synaptic modification in neural networks induced by patterned stimulation. Nature 401:792-796.

Bland BH, Konopacki J, Dyck R (2005) Heterogeneity among hippocampal pyramidal neurons revealed by their relation to theta-band oscillation and synchrony. Exp Neurol 195:458-474.
Cassenaer S, Laurent G (2007) Hebbian STDP in mushroom bodies facilitates the synchronous flow of olfactory information in locusts. Nature 448:709-713.

Chao TC, Chen CM (2005) Learning-induced synchronization and plasticity of a developing neural network. J Comput Neurosci 19:311-324.

Cossart R, Aronov D, Yuste R (2003) Attractor dynamics of network UP states in the neocortex. Nature 423:283-288.

Davison ML (1983) Introduction to multidimensional scaling and its applications. Appl Psychol Meas 7:373-379.

Elbert T, Ray WJ, Kowalik ZJ, Skinner JE, Graf KE, Birbaumer N (1994) Chaos and physiology: deterministic chaos in excitable cell assemblies. Physiol Rev 74:1-47.

Eytan D, Marom S (2006) Dynamics and effective topology underlying synchronization in networks of cortical neurons. J Neurosci 26:8465-8476.

Eytan D, Brenner N, Marom S (2003) Selective adaptation in networks of cortical neurons. J Neurosci 23:9349-9356.

Feller MB, Butts DA, Aaron HL, Rokhsar DS, Shatz CJ (1997) Dynamic processes shape spatiotemporal properties of retinal waves. Neuron 19:293-306.

Foster DJ, Wilson MA (2006) Reverse replay of behavioural sequences in hippocampal place cells during the awake state. Nature 440:680-683.

Fujisawa S, Yamada MK, Nishiyama N, Matsuki N, Ikegaya Y (2004) BDNF boosts spike fidelity in chaotic neural oscillations. Biophys J 86:1820-1828.

Gerstner W, Kistler W (2002) Spiking neuron models: single neurons, populations, plasticity. Cambridge, UK: Cambridge UP. 
Girvan M, Newman ME (2002) Community structure in social and biological networks. Proc Natl Acad Sci U S A 99:7821-7826.

Gütig R, Sompolinsky H (2006) The tempotron: a neuron that learns spike timing-based decisions. Nat Neurosci 9:420-428.

Hosaka R, Araki O, Ikeguchi T (2008) STDP provides the substrate for igniting synfire chains by spatiotemporal input patterns. Neural Comput 20:415-435.

Ikegaya Y, Aaron G, Cossart R, Aronov D, Lampl I, Ferster D, Yuste R (2004) Synfire chains and cortical songs: temporal modules of cortical activity. Science 304:559-564.

Izhikevich EM (2006) Polychronization: computation with spikes. Neural Comput 18:245-282.

Jimbo Y, Tateno T, Robinson HP (1999) Simultaneous induction of pathway-specific potentiation and depression in networks of cortical neurons. Biophys J 76:670-678.

Jun JK, Jin DZ (2007) Development of neural circuitry for precise temporal sequences through spontaneous activity, axon remodeling, and synaptic plasticity. PLoS ONE 2:e723.

Koene R, Hasselmo M (2008) Reversed and forward buffering of behavioral spike sequences enables retrospective and prospective retrieval in hippocampal regions CA3 and CA1. Neural Netw 21:276-288.

Kube K, Herzog A, Michaelis B, de Lima A, Voigt T (2008) Spike-timingdependent plasticity in small-world networks. Neurocomputing 71:1694-1704.

Lee AK, Wilson MA (2002) Memory of sequential experience in the hippocampus during slow wave sleep. Neuron 36:1183-1194.

Marom S, Shahaf G (2002) Development, learning and memory in large random networks of cortical neurons: lessons beyond anatomy. Q Rev Biophys 35:63-87.

Masquelier T, Guyonneau R, Thorpe SJ (2008) Spike timing dependent plasticity finds the start of repeating patterns in continuous spike trains. PLoS ONE 3:e1377.

Masuda N, Kori H (2007) Formation of feedforward networks and frequency synchrony by spike-timing-dependent plasticity. J Comput Neurosci 22:327-345.

Melloni L, Molina C, Pena M, Torres D, Singer W, Rodriguez E (2007)
Synchronization of neural activity across cortical areas correlates with conscious perception. J Neurosci 27:2858-2865.

Mikkonen JE, Grönfors T, Chrobak JJ, Penttonen M (2002) Hippocampus retains the periodicity of gamma stimulation in vivo. J Neurophysiol 88:2349-2354.

Murthy VN, Sejnowski TJ, Stevens CF (1997) Heterogeneous release properties of visualized individual hippocampal synapses. Neuron 18:599-612.

Nowotny T, Zhigulin VP, Selverston AI, Abarbanel HD, Rabinovich MI (2003) Enhancement of synchronization in a hybrid neural circuit by spike-timing dependent plasticity. J Neurosci 23:9776-9785.

Rabinovich MI, Abarbanel HD (1998) The role of chaos in neural systems. Neuroscience 87:5-14.

Raichle ME (2006) Neuroscience. The brain's dark energy. Science 314:1249-1250.

Ramón F, Gronenberg W (2005) Electrical potentials indicate stimulus expectancy in the brains of ants and bees. Cell Mol Neurobiol 25:313-327.

Riehle A, Grün S, Diesmann M, Aertsen A (1997) Spike synchronization and rate modulation differentially involved in motor cortical function. Science 278:1950-1953.

Skinner FK, Chung JY, Ncube I, Murray PA, Campbell SA (2005) Using heterogeneity to predict inhibitory network model characteristics. J Neurophysiol 93:1898-1907.

Sporns O (2002) Graph theory methods for the analysis of neural connectivity patterns. In: Neuroscience databases (Kötter R, ed), pp 171-186. Boston: Klüwer.

Suri RE, Sejnowski TJ (2002) Spike propagation synchronized by temporally asymmetric Hebbian learning. Biol Cybern 87:440-445.

Thivierge JP, Rivest F, Monchi O (2007) Spiking neurons, dopamine, and plasticity: timing is everything, but concentration also matters. Synapse 61:375-390.

Tsuda I (2001) Toward an interpretation of dynamic neural activity in terms of chaotic dynamical systems. Behav Brain Sci 24:793-848.

Yoshioka M (2002) Spike-timing-dependent learning rule to encode spatiotemporal patterns in a network of spiking neurons. Phys Rev E Stat Nonlin Soft Matter Phys 65:011903. 